\title{
Integrated photometric characteristics of galactic open star clusters
}

\author{
S. Lata ${ }^{1}$, A. K. Pandey ${ }^{1}$, R. Sagar ${ }^{1,2}$, and V. Mohan ${ }^{1}$ \\ 1 State Observatory, Manora peak, Nainital 263129, Uttaranchal, India \\ e-mail: sneh, pandey, sagar, vijay@upso.ernet.in \\ 2 Indian Institute of Astrophysics, Bangalore 560034, India
}

Received 19 November 2001 / Accepted 19 February 2002

\begin{abstract}
Integrated $U B V R I$ photometric parameters of 140 galactic open clusters have been computed. Integrated $I(V-R)_{0}$ and $I(V-I)_{0}$ colours as well as integrated parameters for 71 star clusters have been obtained for the first time. These, in combination with published data, altogether 352 objects, are used to study the integrated photometric characteristics of the galactic open clusters. The $I\left(M_{V}\right)$ values range from -9.0 to -1.0 mag corresponding to a range in total mass of the star clusters from $\sim 25$ to $4 \times 10^{4} M_{\odot}$. The integrated colours have a relatively narrow range, e.g., $I(B-V)_{0}$ varies from -0.4 to $1.2 \mathrm{mag}$. The scatter in integrated colours at a given integrated magnitude can be understood in terms of differences in fraction of red giants/supergiants in the clusters. The observed integrated magnitudes and colours agree with the synthetic ones, except the dependences of $I(V-R)_{0}$ and $I(V-I)_{0}$ colours for clusters younger than $\sim 100$ Myrs and also of the integrated magnitudes of oldest clusters. The large sample provides the most accurate age dependence of integrated magnitudes and colours determined so far. The luminosity function of the $I\left(M_{V}\right)$ has a peak around -3.5 mag and its slope indicates that only $\sim 1 \%$ of the open clusters in the galactic disc are brighter than $I\left(M_{V}\right)=-11$ mag. No variation has been found of integrated magnitude with galactocentric distance and metallicity.
\end{abstract}

Key words. galaxies - open clusters - integrated photometry - star formation

\section{Introduction}

Star clusters in a galaxy play an important role in understanding the processes of star formation and stellar evolution in the galaxy as well as galactic structure and evolution. The physical quantities required for such studies are best determined from the observations of individual cluster members. Unfortunately, such observations are not possible in the case of extra-galactic clusters because ground based optical telescopes can not resolve member stars that are separated by less than 1 arcsec. Even with the Hubble Space Telescope, where resolution is improved to 0.1 arcsec, all cluster members cannot be observed in galaxies like M 31, M 33 etc. On the other hand, in such cases integrated photometric parameters can still be observable and, in fact, integrated colours and spectra will remain for long time to come the only way to investigate the evolutionary history of stellar systems beyond the local group of galaxies. To interpret these parameters in terms of age, stellar content, metallicity etc. of the extra-galactic star clusters, it is necessary to study the integrated photometric parameters of those star clusters of our galaxy

Send offprint requests to: S. Lata,

e-mail: sneh@upso.ernet.in where observations of individual cluster members provide these parameters. Without understanding these integrated parameters, it may not be possible to study the observations of integrated light of star clusters of other galaxies, wherein it is not practical even to visually separate out the open clusters from the globulars. Integrated light characteristics of star clusters are thus very important.

Based on the observations of individual stars of a galactic star cluster, integrated photometric parameters have been estimated by several authors (Gray 1965, 1967; Schmidt-Kaler 1967; Piskunov 1972, 1974; Sagar et al. 1983; Spassova \& Baev 1985; Pandey et al. 1989; Battinelli et al. 1994 and references therein). The luminosity distribution of integrated magnitudes of galactic clusters have been discussed in detail by van den Bergh \& Lafontaine (1984). Spassova \& Baev (1985) and Bhatt et al. (1991) have also obtained the integrated luminosity function. The slope of the luminosity function obtained by Bhatt et al. (1991) is steeper than that of van den Bergh \& Lafontaine (1984).

Out of over 1200 open star clusters in our galaxy, reliable integrated photometric parameters are available only for about 200 objects. During the last decade multicolour CCD photometric data have been 

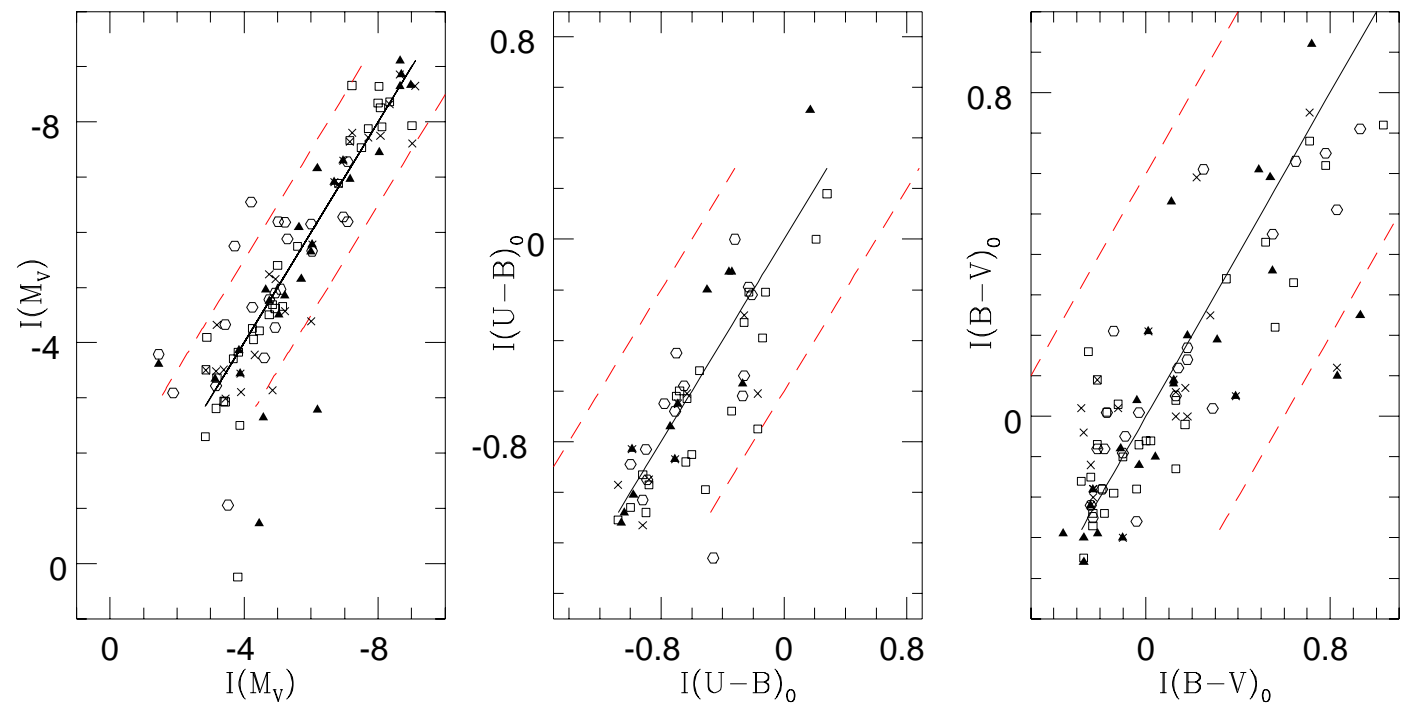

Fig. 1. Comparison of the integrated magnitudes $I\left(M_{V}\right)$ and $I(B-V)_{0}, I(U-B)_{0}$ colours obtained in this work with those given by Sagar et al. (1983) (squares), Pandey et al. (1989) (triangles), Spassova \& Baev (1985) (crosses), and Battinelli et al. (1994) (hexagons). The solid line represents a slope of unity. The dashed lines represent $3 \sigma$ uncertainties in the estimation of parameters.

obtained for a number of unstudied clusters. Mermilliod (1995) maintains a data base WEBDA (http://obswww.unige.ch/webda) for open star clusters. In order to re-investigate the luminosity function studies we have calculated integrated magnitudes and colours of 140 clusters in the $U B V R I$ passbands using the method given by Gray (1965). These in combination with earlier estimates provide integrated photometric parameters for 352 star clusters. The sample has been used to study the integrated luminosity function as well as various photometric properties of integrated light. The data and reduction procedures along with accuracy of parameters are described in the next section.

\section{The data and their comparison}

The $U B V$ Johnson and $R I$ Cousins CCD data of the open clusters have been taken from the WEBDA data base. To calculate the integrated parameters of the clusters, the photometric membership criterion has been used to reduce the effects of field star contamination. For this purpose, we fitted the theoretical isochrones by Bertelli et al. (1994) to the cluster sequence in the colour-magnitude diagram. The isochrones were brightened by $0.75 \mathrm{mag}$ to take into account the maximum effects of binaries present in the cluster. This accounts for the broadening of the mainsequence which may also be due to other reasons, e.g., the photometric errors, internal reddening and spread in metallicity. The stars lying along the cluster sequence were considered members and used to compute the integrated parameters. Wherever CCD data for the bright stars were not available, the photoelectric data for these stars were taken from the data base. Altogether integrated parameters of 140 clusters were determined. The integrated parameters of 71 star clusters have been computed for the first time. The following relation was used to compute integrated magnitudes in $U B V R I$ passbands

$I(m)=-2.5 \log \left[\sum_{i}\left(10^{-0.4 m_{i}}\right)\right]$,

where $m_{i}$ is the magnitude of the $i$ th member star in a passband. In order to account for un-observed faint stars we followed the method suggested by Battinelli et al. (1994). The integrated apparent colours of the clusters are determined as:

$$
\begin{aligned}
I(B-V) & =I(B)-I(V) \\
I(U-B) & =I(U)-I(B) \\
I(V-R) & =I(V)-I(R) \\
I(V-I) & =I(V)-I(I) .
\end{aligned}
$$

The apparent integrated magnitudes and colours, thus obtained, are converted to intrinsic ones and are listed in Table 1 along with the used distance and $E(B-V)$ values taken from the data base. The colour excesses $E(U-B)$, $E(U-V), E(V-R)$ and $E(V-I)$ have been calculated from $E(B-V)$ using the relations $E(U-B)=$ $0.72 E(B-V)+0.05 E(B-V)^{2} ; E(U-V)=1.72 E(B-V)$; $E(V-R)=0.60 E(B-V)$ and $E(V-I)=1.25 E(B-V)$. The possible source of errors in determination of the integrated parameters are same as described by Sagar et al. (1983). The uncertainty in integrated absolute magnitude could be $\pm 0.5 \mathrm{mag}$ and in colours it could be $\pm 0.2 \mathrm{mag}$. Battinelli et al. (1994) have also reported the same order of uncertainty in their estimation of integrated parameters.

Figure 1 shows a comparison of the present integrated values with those available in the literature, where the solid line has a slope of unity. It indicates that the integrated magnitudes $I\left(M_{V}\right)$ are, in general $(\sim 92 \%$ lies 
Table 1. Integrated parameters of the open clusters. The distance modulus $(m-M), E(B-V)$ and log(age) are taken from data base.

\begin{tabular}{|c|c|c|c|c|c|c|c|c|}
\hline \multirow[b]{2}{*}{$\begin{array}{l}\text { Name of } \\
\text { cluster }\end{array}$} & \multirow[b]{2}{*}{$\begin{array}{c}(m-M) \\
(\mathrm{mag})\end{array}$} & \multirow[b]{2}{*}{$\begin{array}{c}E(B-V) \\
\quad(\mathrm{mag})\end{array}$} & \multirow[b]{2}{*}{$\begin{array}{c}\log t \\
(t \text { in years })\end{array}$} & \multicolumn{5}{|c|}{ Integrated Absolute values } \\
\hline & & & & $\begin{array}{c}\left(M_{V}\right) \\
(\mathrm{mag})\end{array}$ & $\begin{array}{c}I(U-V)_{0} \\
(\mathrm{mag})\end{array}$ & $\begin{array}{c}I(B-V)_{0} \\
(\mathrm{mag})\end{array}$ & $\begin{array}{c}I(V-R)_{0} \\
\quad(\mathrm{mag})\end{array}$ & $\begin{array}{c}I(V-I)_{0} \\
(\mathrm{mag})\end{array}$ \\
\hline 1 & 2 & 3 & 4 & 5 & 6 & 7 & 8 & 9 \\
\hline NGC 129 & 12.71 & 0.55 & 7.76 & -6.03 & -0.25 & 0.39 & - & - \\
\hline NGC 146 & 13.61 & 0.49 & 7.60 & -4.88 & -0.75 & -0.07 & - & - \\
\hline NGC 188 & 11.41 & 0.09 & 9.82 & -2.86 & - & 1.13 & 0.7 & 1.39 \\
\hline NGC 381 & 11.27 & 0.34 & 8.47 & -2.98 & -0.31 & 0.11 & - & - \\
\hline NGC 433 & 14.58 & 0.86 & 7.50 & -4.60 & -0.36 & -0.09 & - & - \\
\hline NGC 436 & 13.88 & 0.48 & 7.78 & -5.21 & -0.14 & 0.04 & - & 0.13 \\
\hline NGC 457 & 13.77 & 0.48 & 7.15 & -9.01 & -0.04 & 0.13 & - & -0.11 \\
\hline NGC 581 & 13.16 & 0.44 & 7.13 & -6.82 & -0.75 & -0.12 & - & -0.10 \\
\hline NGC 637 & 14.12 & 0.70 & 6.96 & -5.47 & -1.13 & -0.28 & - & - \\
\hline NGC 654 & 14.64 & 0.85 & 7.08 & -7.71 & -0.47 & -0.21 & - & - \\
\hline NGC 659 & 14.00 & 0.64 & 7.63 & -5.00 & -0.26 & -0.14 & - & - \\
\hline NGC 663 & 14.42 & 0.82 & 7.13 & -8.02 & -0.74 & -0.14 & - & - \\
\hline NGC 869 & 13.48 & 0.58 & 7.10 & -8.65 & -1.22 & -0.23 & - & - \\
\hline NGC 884 & 13.83 & 0.58 & 7.15 & -8.65 & -0.68 & 0.01 & - & - \\
\hline NGC 1039 & 8.82 & 0.10 & 8.26 & -3.19 & - & 0.17 & - & - \\
\hline NGC 1193 & 13.55 & 0.12 & 9.90 & -3.00 & - & 1.06 & - & - \\
\hline NGC 1245 & 13.07 & 0.31 & 9.16 & -3.60 & - & 0.63 & - & - \\
\hline NGC 1750 & 10.08 & 0.34 & 8.30 & -3.46 & -0.40 & 0.04 & 0.05 & 0.13 \\
\hline NGC 1798 & 14.75 & 0.51 & 9.15 & -4.86 & 0.97 & 0.82 & - & 1.14 \\
\hline NGC 1893 & 14.51 & 0.49 & 7.00 & -7.63 & -0.79 & -0.19 & - & - \\
\hline NGC 1907 & 12.24 & 0.43 & 8.61 & -6.19 & - & 0.93 & - & - \\
\hline NGC 1912 & 11.07 & 0.26 & 8.48 & -5.22 & - & 0.83 & - & 0.32 \\
\hline NGC 1931 & 13.40 & 0.58 & 7.13 & -3.15 & -1.02 & -0.20 & -0.05 & -0.07 \\
\hline NGC 2112 & 11.12 & 0.50 & 9.48 & -1.88 & - & 0.65 & - & - \\
\hline NGC 2158 & 14.51 & 0.49 & 9.27 & -2.81 & - & 0.61 & - & - \\
\hline NGC 2168 & 10.44 & 0.25 & 8.00 & -4.93 & -0.10 & 0.13 & - & 0.06 \\
\hline NGC 2192 & 13.36 & 0.20 & 9.04 & -3.53 & 0.59 & 0.49 & - & 0.54 \\
\hline NGC 2204 & 13.44 & 0.08 & 9.27 & -4.65 & - & 0.72 & - & 1.10 \\
\hline NGC 2243 & 13.17 & 0.03 & 9.49 & -2.67 & - & 0.23 & - & 0.78 \\
\hline NGC 2244 & 12.61 & 0.48 & 6.80 & -6.69 & -1.07 & -0.24 & - & - \\
\hline NGC 2264 & 9.57 & 0.06 & 6.99 & -5.29 & -1.27 & -0.27 & -0.11 & -0.24 \\
\hline NGC 2266 & 12.98 & 0.10 & 8.86 & -3.83 & 1.02 & 0.62 & - & - \\
\hline NGC 2355 & 12.10 & 0.12 & 8.85 & -3.67 & 0.81 & 0.59 & - & 0.64 \\
\hline NGC 2383 & 12.33 & 0.27 & 7.61 & -3.18 & - & -0.05 & 0.04 & 0.08 \\
\hline NGC 2384 & 12.95 & 0.29 & 6.92 & -5.63 & - & -0.21 & -0.22 & -0.01 \\
\hline NGC 2420 & 12.02 & 0.02 & 9.32 & -3.44 & - & 0.71 & - & - \\
\hline
\end{tabular}

within $3 \sigma$ ), in fair agreement barring some points that deviate considerably from the relation. The difference is mainly due to the difference in the values of distance modulus used. The $I(U-B)_{0}$ and $I(B-V)_{0}$ colours are also in fair agreement ( $\sim 97 \%$ lies within $3 \sigma$ ) barring a few points which show large deviation.

\section{Comparison of integrated parameters with theoretical models}

In the present work we have calculated integrated photometric parameters for 140 clusters. The integrated photometric data along with age estimates for another 162 clusters are taken from Sagar et al. (1983) and
Pandey et al. (1989). When age estimates are not available in these catalogues they are taken from the catalogue of Mermilliod (1995). As a comparison of these observed integrated parameters with those obtained for synthetic clusters provides a check to basic assumptions made to derive synthetic parameters, the same is done below.

\subsection{Colour-magnitude diagram}

In Fig. 2, we plot $I\left(M_{V}\right)$ against $I(B-V)_{0}$. The values of $I\left(M_{V}\right)$ range from -9.0 to $-1.0 \mathrm{mag}$ while $I(B-V)_{0}$ varies from -0.4 to $1.2 \mathrm{mag}$. The variations in the parameters are thus significantly larger than the corresponding errors in them. The $I\left(M_{V}\right)$ is related linearly with the 
Table 1. continued.

\begin{tabular}{|c|c|c|c|c|c|c|c|c|}
\hline \multirow{3}{*}{$\begin{array}{c}\text { Name of } \\
\text { cluster } \\
1\end{array}$} & \multirow{3}{*}{$\begin{array}{c}(m-M) \\
(\mathrm{mag}) \\
2\end{array}$} & \multirow{3}{*}{$\begin{array}{c}E(B-V) \\
(\mathrm{mag}) \\
3\end{array}$} & \multirow{3}{*}{$\begin{array}{c}\log t \\
(t \text { in years }) \\
4\end{array}$} & \multicolumn{5}{|c|}{$\begin{array}{l}\text { Integrated Absolute Values } \\
\end{array}$} \\
\hline & & & & $\begin{array}{c}I\left(M_{V}\right) \\
(\mathrm{mag})\end{array}$ & $\begin{array}{c}I(U-V)_{0} \\
(\mathrm{mag})\end{array}$ & $\begin{array}{c}I(B-V)_{0} \\
(\mathrm{mag})\end{array}$ & $\begin{array}{c}I(V-R)_{0} \\
\quad(\mathrm{mag})\end{array}$ & $\begin{array}{c}I(V-I)_{0} \\
\quad(\mathrm{mag})\end{array}$ \\
\hline & & & & 5 & 6 & 7 & 8 & 9 \\
\hline NGC 2421 & 13.23 & 0.50 & 7.25 & -4.75 & -0.89 & -0.21 & $\overline{-}$ & $\overline{-}$ \\
\hline NGC 2439 & 14.39 & 0.39 & 7.13 & -8.00 & 0.38 & 0.52 & - & - \\
\hline NGC 2453 & 13.53 & 0.47 & 7.00 & -5.15 & - & -0.25 & - & -0.12 \\
\hline NGC 2477 & 12.00 & 0.33 & 8.95 & -5.70 & 0.66 & 0.49 & - & 0.67 \\
\hline NGC 2489 & 13.25 & 0.45 & 8.18 & -3.91 & 1.06 & 0.35 & - & - \\
\hline NGC 2506 & 12.89 & 0.13 & 9.07 & -4.31 & 0.76 & 0.54 & 0.37 & 0.78 \\
\hline NGC 2567 & 11.47 & 0.09 & 8.43 & -3.68 & - & 0.56 & - & - \\
\hline NGC 2627 & 14.62 & 0.63 & 8.20 & -4.84 & - & 0.24 & - & 0.00 \\
\hline NGC 2658 & 14.18 & 0.40 & 8.30 & -3.87 & -0.03 & 0.03 & - & - \\
\hline NGC 2660 & 13.52 & 0.38 & 9.22 & -4.08 & 0.95 & 0.61 & - & 1.31 \\
\hline NGC 2670 & 11.63 & 0.45 & 7.73 & -4.24 & - & 0.62 & - & 0.74 \\
\hline NGC 2682 & 9.75 & 0.07 & 9.72 & -3.16 & 1.06 & 0.78 & 0.56 & - \\
\hline NGC 2818 & 13.11 & 0.18 & 8.97 & -3.54 & 0.61 & 0.48 & 0.17 & 0.37 \\
\hline NGC 2910 & 11.16 & 0.07 & 8.50 & -3.14 & - & 0.12 & - & - \\
\hline NGC 3114 & 9.95 & 0.06 & 7.93 & -5.33 & - & 0.22 & - & - \\
\hline NGC 3766 & 11.98 & 0.20 & 7.42 & -7.09 & -0.53 & 0.11 & - & -0.14 \\
\hline NGC 4103 & 12.16 & 0.29 & 7.46 & -4.70 & -0.82 & -0.18 & -0.04 & -0.03 \\
\hline NGC 4755 & 12.90 & 0.40 & 7.26 & -8.65 & -0.85 & -0.11 & -0.05 & -0.03 \\
\hline NGC 4815 & 14.64 & 0.78 & 8.48 & -6.02 & 0.32 & 0.29 & - & - \\
\hline NGC 5168 & 13.34 & 0.66 & 8.18 & -3.72 & 0.52 & 0.33 & - & - \\
\hline NGC 5460 & 9.23 & 0.12 & 8.06 & -3.84 & - & 0.04 & - & - \\
\hline NGC 5606 & 12.98 & 0.49 & 6.87 & -5.02 & -1.14 & -0.24 & -0.10 & -0.22 \\
\hline NGC 5617 & 12.57 & 0.51 & 7.57 & -4.25 & -0.87 & -0.17 & - & - \\
\hline NGC 5999 & 13.00 & 0.45 & 8.60 & -3.85 & - & 0.18 & - & 0.29 \\
\hline NGC 6005 & 13.59 & 0.45 & 9.08 & -3.66 & - & 0.45 & - & 0.80 \\
\hline NGC 6031 & 12.75 & 0.50 & 8.40 & -3.87 & - & -0.03 & - & 0.00 \\
\hline NGC 6067 & 12.34 & 0.37 & 7.97 & -6.18 & - & 0.31 & - & 0.61 \\
\hline NGC 6087 & 10.26 & 0.18 & 7.91 & -5.09 & -0.03 & 0.18 & - & - \\
\hline NGC 6192 & 12.94 & 0.66 & 7.96 & -4.57 & -0.46 & -0.10 & - & - \\
\hline NGC 6204 & 11.63 & 0.43 & 7.92 & -3.75 & -0.58 & -0.07 & - & - \\
\hline NGC 6231 & 12.30 & 0.41 & 6.75 & -8.34 & -1.15 & -0.23 & - & -0.18 \\
\hline NGC 6253 & 11.53 & 0.20 & 9.70 & -2.68 & 1.15 & 0.81 & 0.58 & 0.97 \\
\hline NGC 6451 & 13.55 & 0.67 & 8.05 & -4.45 & -0.23 & 0.11 & - & 0.52 \\
\hline NGC 6475 & 7.08 & 0.06 & 8.11 & -3.89 & - & 0.12 & - & 0.50 \\
\hline NGC 6520 & 13.34 & 0.44 & 8.14 & -5.03 & -0.54 & -0.04 & - & - \\
\hline
\end{tabular}

total mass of the cluster and according to Lyngå (1982), $I\left(M_{V}\right) \sim-8 \mathrm{mag}$ correspond to $4 \times 10^{3} M_{\odot}$. Part of the variation in integrated parameters of the clusters can be understood in terms of the difference in the total mass of the clusters. A comparison of the observed data points with the Battinelli \& Capuzzo-Dolcetta (1989) theoretical evolutionary model for solar composition indicates that range in total mass of the present sample of clusters is from $\sim 25$ to $4 \times 10^{4} M_{\odot}$. The scatter in $I(B-V)_{0}$ at a given $I\left(M_{V}\right)$ increases with decreasing integrated brightness of the cluster. This can be understood if one considers the fraction $f$ of red giants/supergiants present in the cluster. The fraction is calculated for the three brightest bins of $V$ magnitude assuming that the brightest bins are not affected by the incompleteness of the data. For a given
$I\left(M_{V}\right)$ (or total mass) of the cluster, the value of $f$ will depend upon the age, the mass function (MF) and dynamical state of the cluster and consequently the $I(B-V)_{0}$ value increases with the cluster age. On the other hand, for star clusters of the same age and $I\left(M_{V}\right)$, but differing $\mathrm{MF}$ and the dynamical evolution, the integrated colours of those open clusters will be relatively redder in which relatively heavier stars are present since, due to evolution, the stars will become red giants/supergiants and will therefore affect the $I(B-V)_{0}$. Figure 2, in fact, provides an observational support for this, as the clusters with a large fraction of red giants/supergiants have relatively redder $I(B-V)_{0}$ values. The spread in the values of $I\left(M_{V}\right)$ and $I(B-V)_{0}$ present in Fig. 2 can thus be understood in terms of above mentioned factors. 
Table 1. continued.

\begin{tabular}{|c|c|c|c|c|c|c|c|c|}
\hline \multirow{3}{*}{$\begin{array}{l}\text { Name of } \\
\text { cluster } \\
1\end{array}$} & \multirow{3}{*}{$\begin{array}{c}(m-M) \\
(\mathrm{mag}) \\
2\end{array}$} & \multirow{3}{*}{$\begin{array}{c}E(B-V) \\
(\mathrm{mag}) \\
3\end{array}$} & \multirow{3}{*}{$\begin{array}{c}\log t \\
(t \text { in years }) \\
4\end{array}$} & \multicolumn{5}{|c|}{$\begin{array}{l}\text { Integrated Absolute Values } \\
\end{array}$} \\
\hline & & & & $\begin{array}{c}I\left(M_{V}\right) \\
(\mathrm{mag})\end{array}$ & $\begin{array}{c}I(U-V)_{0} \\
(\mathrm{mag})\end{array}$ & $\begin{array}{c}I(B-V)_{0} \\
(\mathrm{mag})\end{array}$ & $\begin{array}{c}I(V-R)_{0} \\
\quad(\mathrm{mag})\end{array}$ & $\begin{array}{c}I(V-I)_{0} \\
(\mathrm{mag})\end{array}$ \\
\hline & & & & 5 & 6 & 7 & 8 & 9 \\
\hline NGC 6603 & 15.31 & 0.79 & 8.30 & -6.40 & - & 0.22 & $\overline{-}$ & 0.26 \\
\hline NGC 6611 & 14.03 & 0.76 & 6.10 & -7.15 & -1.36 & -0.28 & - & - \\
\hline NGC 6649 & 16.01 & 1.37 & 7.69 & -6.92 & -0.34 & 0.08 & - & - \\
\hline NGC 6705 & 12.61 & 0.42 & 8.05 & -6.00 & -0.43 & 0.18 & - & - \\
\hline NGC 6709 & 10.90 & 0.32 & 8.01 & -3.43 & - & 0.11 & - & 0.51 \\
\hline NGC 6716 & 9.51 & 0.13 & 7.97 & -2.84 & -0.32 & 0.00 & - & - \\
\hline NGC 6755 & 13.69 & 0.85 & 7.78 & -4.21 & -0.70 & -0.14 & - & - \\
\hline NGC 6791 & 13.93 & 0.20 & 9.86 & -4.14 & 1.84 & 1.02 & - & 1.45 \\
\hline NGC 6823 & 14.40 & 0.85 & 6.56 & -7.22 & -1.12 & -0.24 & -0.16 & -0.27 \\
\hline NGC 6871 & 12.41 & 0.47 & 6.95 & -7.28 & -1.13 & -0.24 & - & - \\
\hline NGC 6913 & 13.47 & 0.78 & 7.19 & -6.96 & -0.81 & -0.10 & - & - \\
\hline NGC 7044 & 14.63 & 0.70 & 9.20 & -3.93 & 1.06 & 0.58 & - & 0.82 \\
\hline NGC 7380 & 14.20 & 0.60 & 6.96 & -7.50 & -0.69 & -0.18 & - & - \\
\hline NGC 7419 & 17.28 & 1.71 & 7.15 & -6.51 & -0.90 & 0.04 & -0.05 & -0.11 \\
\hline NGC 7510 & 16.03 & 1.02 & 7.00 & -7.69 & - & -0.16 & - & - \\
\hline NGC 7654 & 12.95 & 0.65 & 7.72 & -7.08 & - & 0.14 & - & -0.09 \\
\hline NGC 7762 & 11.74 & 0.76 & 9.23 & -1.45 & - & 0.55 & - & - \\
\hline NGC 7790 & 14.10 & 0.53 & 7.74 & -4.75 & -0.30 & -0.03 & - & - \\
\hline Trum 1 & 14.02 & 0.63 & 7.43 & -5.65 & -0.92 & -0.04 & - & - \\
\hline Trum 5 & 14.24 & 0.58 & 9.50 & -5.06 & - & 0.84 & - & 1.01 \\
\hline Trum 14 & 14.21 & 0.52 & 6.50 & -8.03 & -1.31 & -0.27 & -0.12 & -0.22 \\
\hline Trum 16 & 14.08 & 0.52 & 6.43 & -8.98 & -1.42 & -0.36 & - & - \\
\hline Be 2 & 16.16 & 0.80 & 8.90 & -4.18 & 0.60 & 0.46 & - & - \\
\hline Be 7 & 14.61 & 0.80 & 6.60 & -4.54 & -0.94 & -0.15 & - & - \\
\hline Be 14 & 15.37 & 0.52 & 9.20 & -4.07 & 0.60 & 0.67 & - & 0.51 \\
\hline Be 18 & 14.50 & 0.46 & 9.63 & -4.90 & 2.14 & 0.93 & - & - \\
\hline Be 21 & 15.93 & 0.76 & 9.34 & -3.41 & 1.56 & 0.37 & - & - \\
\hline Be 22 & 16.01 & 0.64 & 9.68 & -3.27 & - & 0.88 & - & - \\
\hline Be 29 & 16.40 & 0.21 & 9.60 & -4.64 & - & 0.70 & - & - \\
\hline Be 30 & 15.00 & 0.50 & 8.48 & -5.11 & 0.21 & 0.29 & - & - \\
\hline Be 31 & 14.25 & 0.13 & 9.90 & -2.57 & 0.95 & 0.73 & - & - \\
\hline Be 32 & 13.20 & 0.16 & 9.53 & -2.97 & 1.06 & 0.78 & - & - \\
\hline Be 33 & 15.57 & 0.70 & 8.85 & -4.61 & - & 0.43 & - & 0.62 \\
\hline Be 39 & 13.78 & 0.12 & 9.90 & -4.28 & - & 0.93 & - & - \\
\hline
\end{tabular}

The integrated colour-magnitude diagram seems to confirm the reliability of cluster-integrated photometry as only just a few lie to the left of the ZAMS. The anomalously blue $\left(I(B-V)_{0}<-0.33\right)$ colours are within the uncertainties in the integrated $I(B-V)_{0}$ colour. As already mentioned by Sagar et al. (1983), contrary to the suggestions made by Gray (1965), the observed as well as theoretical relations between $I\left(M_{V}\right)$ and $I(B-V)_{0}$ do not seem to be linear.

\subsection{Evolutionary effects}

In addition to the data of 302 clusters discussed above, we include the data of 50 additional clusters from Battinelli et al. (1994) for further discussion. They could not be used in Fig. 2 due to lack of knowledge of the fraction of red giants/supergiants in individual clusters. The observed as well as theoretical dependences of the integrated magnitude and colours are plotted against log(age) in Fig. 3. This indicates that as a cluster becomes older, its integrated brightness decreases while the integrated colours become redder, as expected due to stellar evolutionary effects. However, at a given cluster age, there is a scatter in integrated parameters. In $I\left(M_{V}\right)$, the standard deviation $(\sigma)$ in various log (age) bins ranges from 0.9 to $1.7 \mathrm{mag}$ while in integrated colours it ranges from 0.04 to 0.3 mag. The scatter in the integrated magnitudes is thus larger than that in the integrated colours and can not be accounted for in terms of errors in $I\left(M_{V}\right)$. This can be explained in terms of difference in the initial mass of the parent cloud from which a cluster is formed. It can vary from $10^{4}$ to $10^{6} M_{\odot}$, as the star formation efficiency is 
Table 1. continued.

\begin{tabular}{|c|c|c|c|c|c|c|c|c|}
\hline \multirow{3}{*}{$\begin{array}{l}\text { Name of } \\
\text { cluster } \\
1\end{array}$} & \multirow{3}{*}{$\begin{array}{c}(m-M) \\
(\mathrm{mag}) \\
2\end{array}$} & \multirow{3}{*}{$\begin{array}{c}E(B-V) \\
(\mathrm{mag}) \\
3\end{array}$} & \multirow{3}{*}{$\begin{array}{c}\log t \\
(t \text { in years }) \\
4\end{array}$} & \multicolumn{5}{|c|}{ Integrated Absolute Values } \\
\hline & & & & $\begin{array}{c}I\left(M_{V}\right) \\
(\mathrm{mag})\end{array}$ & $\begin{array}{c}I(U-V)_{0} \\
(\mathrm{mag})\end{array}$ & $\begin{array}{c}I(B-V)_{0} \\
(\mathrm{mag})\end{array}$ & $\begin{array}{c}I(V-R)_{0} \\
(\mathrm{mag})\end{array}$ & $\begin{array}{c}I(V-I)_{0} \\
(\mathrm{mag})\end{array}$ \\
\hline & & & & 5 & 6 & 7 & 8 & 9 \\
\hline Be 42 & 12.45 & 0.74 & 9.03 & -0.72 & 0.71 & 0.64 & 0.33 & - \\
\hline Be 54 & 14.27 & 0.77 & 9.60 & -3.21 & - & 0.85 & - & - \\
\hline Be 58 & 14.61 & 0.55 & 8.42 & -5.04 & 0.37 & 0.32 & - & - \\
\hline Be 62 & 14.05 & 0.86 & 7.00 & -4.04 & -0.58 & -0.08 & - & - \\
\hline Be 64 & 16.30 & 1.05 & 9.00 & -4.01 & - & 0.60 & 0.48 & 0.59 \\
\hline Be 69 & 14.60 & 0.65 & 8.95 & -3.16 & 0.59 & 0.38 & 0.15 & 0.42 \\
\hline Be 81 & 15.59 & 1.00 & 9.00 & -4.70 & - & 0.60 & - & - \\
\hline Be 86 & 12.93 & 0.88 & 7.05 & -5.98 & -1.20 & -0.28 & -0.09 & -0.12 \\
\hline Be 99 & 14.41 & 0.30 & 9.50 & -3.66 & - & 1.00 & - & - \\
\hline IC 1311 & 13.93 & 0.28 & 9.20 & -3.90 & 1.17 & 0.85 & - & - \\
\hline IC 1590 & 13.37 & 0.32 & 6.54 & -6.52 & -1.19 & -0.20 & - & - \\
\hline IC 1805 & 14.27 & 0.80 & 6.67 & -8.07 & -1.13 & -0.23 & - & - \\
\hline IC 2602 & 6.23 & 0.05 & 7.36 & -4.28 & - & -0.19 & - & - \\
\hline IC 4665 & 8.29 & 0.19 & 7.58 & -3.40 & - & -0.10 & - & - \\
\hline IC 4651 & 9.90 & 0.13 & 9.52 & -2.89 & - & 1.03 & - & 0.81 \\
\hline IC 4996 & 13.44 & 0.66 & 7.00 & -7.17 & -1.25 & -0.27 & -0.17 & -0.37 \\
\hline Pism 19 & 16.39 & 1.45 & 9.04 & -4.59 & - & 0.45 & - & - \\
\hline Pism 20 & 16.41 & 1.20 & 6.38 & -8.69 & -1.26 & -0.23 & - & - \\
\hline Coll 74 & 13.21 & 0.38 & 9.11 & -2.88 & 0.80 & 0.76 & - & 1.15 \\
\hline Coll 228 & 12.76 & 0.30 & 6.69 & -7.70 & -1.26 & -0.24 & - & - \\
\hline Coll 261 & 12.57 & 0.27 & 9.95 & -2.87 & - & 1.07 & - & 1.05 \\
\hline Coll 272 & 13.25 & 0.45 & 7.11 & -5.31 & -0.75 & -0.16 & -0.01 & 0.01 \\
\hline Haff 6 & 13.94 & 0.43 & 9.00 & -3.81 & - & 0.47 & - & - \\
\hline King 7 & 15.71 & 1.25 & 8.80 & -4.96 & - & 0.25 & - & - \\
\hline King 10 & 16.24 & 1.16 & 7.70 & -5.34 & -0.77 & -0.19 & -0.11 & 0.68 \\
\hline Stock 24 & 13.85 & 0.50 & 8.08 & -5.04 & - & 0.26 & - & - \\
\hline Rup 32 & 15.24 & 0.50 & 7.08 & -5.20 & - & -0.26 & - & - \\
\hline Rup 79 & 14.40 & 0.76 & 7.66 & -4.46 & -0.68 & -0.13 & - & - \\
\hline Rup 115 & 13.75 & 0.65 & 8.78 & -3.50 & - & 0.29 & - & 0.55 \\
\hline Rup 120 & 13.75 & 0.70 & 8.18 & -3.76 & - & 0.14 & - & 0.27 \\
\hline Terzan 7 & 17.48 & 0.12 & 9.89 & -5.20 & - & 0.80 & - & - \\
\hline Melo 71 & 12.25 & 0.01 & 8.65 & -3.83 & 0.85 & 0.64 & - & 0.77 \\
\hline Melo 105 & 13.08 & 0.48 & 8.29 & -4.27 & -0.04 & 0.07 & - & - \\
\hline Dolid 25 & 16.19 & 0.81 & 7.11 & -8.11 & -0.32 & 0.02 & - & - \\
\hline Bochum 2 & 15.65 & 0.86 & 6.56 & -6.05 & -1.43 & -0.30 & - & - \\
\hline
\end{tabular}

generally only a few percent and the observed total stellar mass of the cluster varies from $\sim 25$ to $4 \times 10^{4} M_{\odot}$ (see Sect. 3.1). Since the clusters formed from the massive molecular clouds will contain a larger number of brighter stars in comparison to those clusters which are formed from the less massive molecular clouds, there will be a range in integrated parameters of star clusters of a given age.

The age dependence of the integrated parameters has been discussed by many investigator (see Gray 1965; Sagar et al. 1983; Balázs 1986; Pandey et al. 1989; Battinelli et al. 1994). The least-square linear regressions used in the present sample of 352 yield the following relations between the various parameters:

$I\left(M_{V}\right)=(1.20 \pm 0.08)(\log t)+(-14.12 \pm 0.66)$ with $\chi^{2}=2.017$

$I(U-V)_{0}=(0.74 \pm 0.03)(\log t)+(-6.07 \pm 0.23)$

with $\chi^{2}=0.171$

$I(B-V)_{0}=(0.31 \pm 0.01)(\log t)+(-2.36 \pm 0.09)$

with $\chi^{2}=0.037$

$I(V-R)_{0}=(0.22 \pm 0.02)(\log t)+(-1.65 \pm 0.17)$

with $\chi^{2}=0.011$

$I(V-I)_{0}=(0.44 \pm 0.03)(\log t)+(-3.25 \pm 0.25)$

with $\chi^{2}=0.048$

where $t$ is the age (in years) of the cluster. The above relations indicate that the age of a cluster can be estimated 


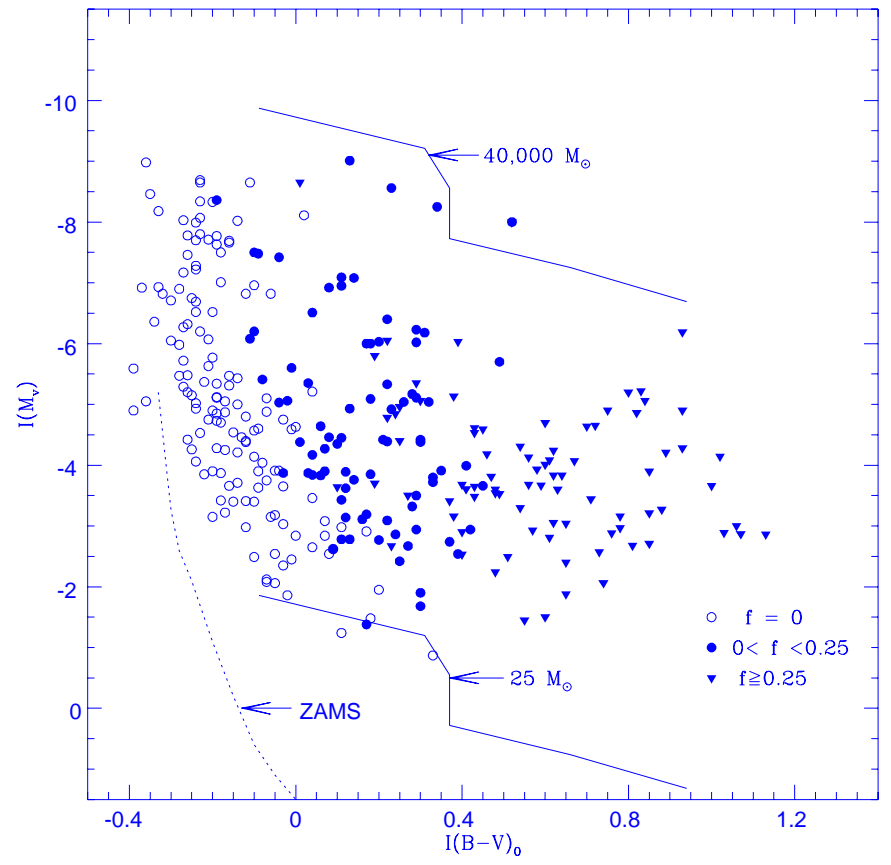

Fig. 2. The $I\left(M_{V}\right), I(B-V)_{0}$ diagram. $f$ is the fraction of red giants/supergiants in the open clusters. The curves with solid lines are theoretical evolutionary tracks given by Battinelli \& Capuzzo-Dolcetta (1989). The dotted curve is the location of the zero-age main sequence (ZAMS) given by Schmidt-Kaler (1982).

from the observed integrated parameters of star clusters of solar metallicity in external galaxies and it can be determined accurately with integrated colours in comparison to integrated magnitudes. Amongst colours, the $I(U-V)_{0}$ colour provides the best age estimate.

A comparison of the coefficients of the relations 6 to 8 with the corresponding values given by Gray (1965) and Balázs (1986) indicates that the present coefficients are better determined due to use of larger sample. In order to see whether higher orders fit the data points better, the least-square fitting of a polynomial of second order has been carried out. They yield the following relation:

$$
\begin{aligned}
I(B-V)_{0}= & (0.07 \pm 0.01)(\log t)^{2} \\
& +(-0.79 \pm 0.16)(\log t)+(1.99 \pm 0.64)
\end{aligned}
$$

with $\chi^{2}=0.033$ indicating a marginal improvement over the $\chi^{2}$ value earlier obtained with the linear relation. Similar improvements are also observed in other relations, e.g., in

$$
\begin{aligned}
I\left(M_{V}\right)= & (-0.36 \pm 0.08)(\log t)^{2}+(6.90 \pm 1.24)(\log t) \\
& +(-36.53 \pm 4.88), \quad \chi^{2}=1.904 .
\end{aligned}
$$

The average value of observed parameters is compared with the the synthetic parameters in Fig. 3. This indicates that:

(i) The theoretical dependences of $I(B-V)_{0}-$ log (age) and $I(U-B)_{0}-\log$ (age), given by Pandey et al. (1989); Maraston (1998) and Brocato et al. (1999) agree fairly

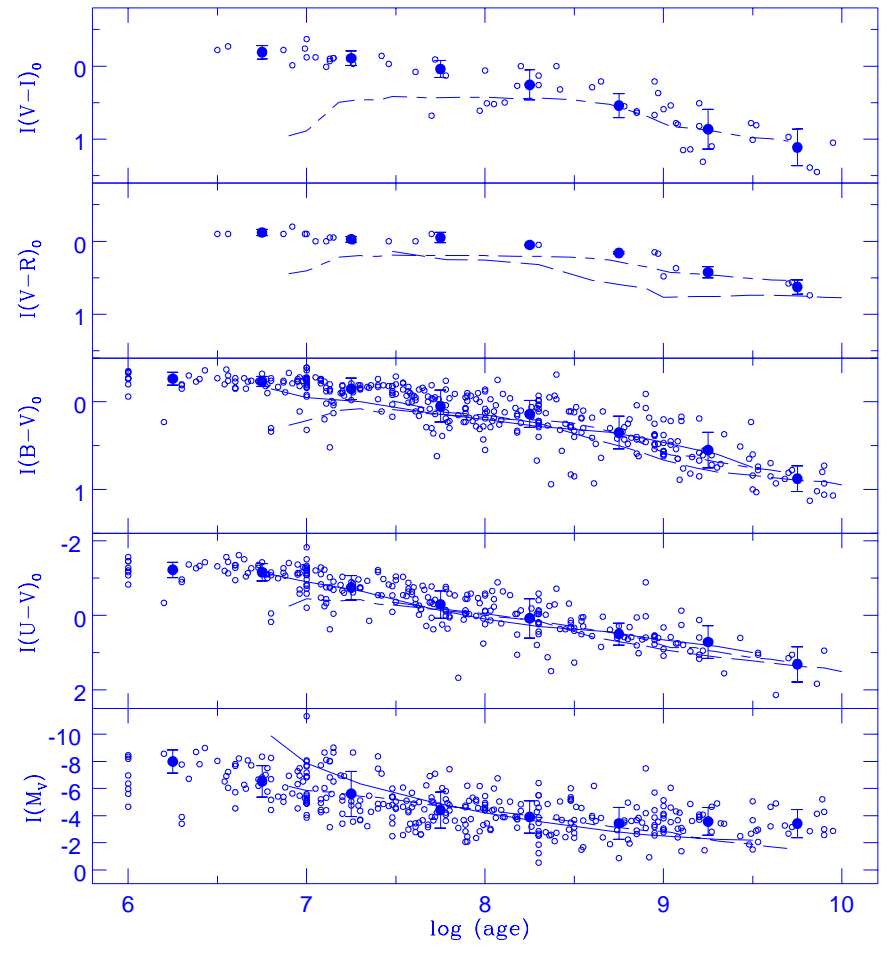

Fig. 3. The $I\left(M_{V}\right)$ and integrated colours are shown as a function of log (age). The curves represent the theoretical dependences predicted by models. Solid line is by Pandey et al. (1989) for whole cluster, long dash line is by Maraston (1998) and short long dash line is by Brocato et al. (1999). The filled circles and error bars are the average and $\sigma$ respectively of the data points binned in $\log ($ age $)$.

well with the observed ones. However, the theoretical dependences of $I(V-R)_{0}$ and $I(V-I)_{0}$ on log (age), given by Maraston (1998) and Brocato et al. (1999) differ from the observed one for clusters younger than $\sim 100$ Myrs.

(ii) The observed dependence of integrated luminosity on the age is compared with models in Fig. 3. The theoretical dependences were obtained for a fixed value of MF barring the work of Pandey et al. (1989) who assumed a time-dependent initial mass function (IMF). The comparison indicates that a steeper MF is needed to match the integrated luminosities of oldest clusters (age $\geq 10^{9}$ years). Elson \& Fall (1985) reported that, unlike the relation between colour and age, the relation between luminosity and age depends strongly on the IMF.

\section{Luminosity distribution of open clusters}

Figure 4 shows the integrated absolute magnitude distribution of 352 clusters according to their ages which indicates a maximum at $I\left(M_{V}\right)=-3.5 \mathrm{mag}$ and a sharp cut off at $I\left(M_{V}\right)=-2.0 \mathrm{mag}$ which agree with the results earlier obtained by van den Bergh \& Lafontaine (1984) and Bhatt et al. (1991). Van den Bergh \& Lafontaine (1984) concluded that the sharp cut-off at $I\left(M_{V}\right)=-2.0$ mag is 
Table 2. Completeness radius and surface density for the different magnitude intervals.

\begin{tabular}{|c|c|c|c|c|c|c|c|}
\hline \multicolumn{3}{|c|}{$\begin{array}{c}I\left(M_{V}\right) \\
\text { interval } \\
(\mathrm{mag})\end{array}$} & $\begin{array}{r}\text { Total } \\
\text { number of } \\
\text { clusters }\end{array}$ & $\begin{array}{r}\text { Clusters } \\
\text { within } \\
3.0 \mathrm{kpc}\end{array}$ & $\begin{array}{c}\text { Completeness } \\
\text { radius, } r \\
(\mathrm{kpc})\end{array}$ & $\begin{array}{c}\text { Number of } \\
\text { clusters } \\
\text { within } r\end{array}$ & $\begin{array}{c}\text { Surface } \\
\text { density, } \sigma \\
\left(\text { cluster } / \mathrm{kpc}^{2} \text { ) }\right.\end{array}$ \\
\hline 2 & to & 1 & 1 & 1 & - & - & - \\
\hline 1 & to & 0 & 0 & 0 & - & - & - \\
\hline 0 & to & -1 & 4 & 4 & - & - & - \\
\hline-1 & to & -2 & 10 & 9 & - & - & - \\
\hline-2 & to & -3 & 47 & 48 & 2.00 & 40 & 3.18 \\
\hline-3 & to & -4 & 81 & 68 & 2.51 & 63 & 3.18 \\
\hline-4 & to & -5 & 78 & 62 & 3.16 & 65 & 2.07 \\
\hline-5 & to & -6 & 56 & 45 & 3.98 & 50 & 1.01 \\
\hline-6 & to & -7 & 33 & 20 & 5.01 & 28 & 0.36 \\
\hline-7 & to & -8 & 21 & 15 & 6.61 & 21 & 0.15 \\
\hline-8 & to & -9 & 19 & 13 & 6.92 & 18 & 0.12 \\
\hline-9 & to & -10 & 1 & 0 & - & - & - \\
\hline-10 & to & -11 & 0 & 0 & - & - & - \\
\hline-11 & to & -12 & 1 & 0 & - & - & - \\
\hline
\end{tabular}

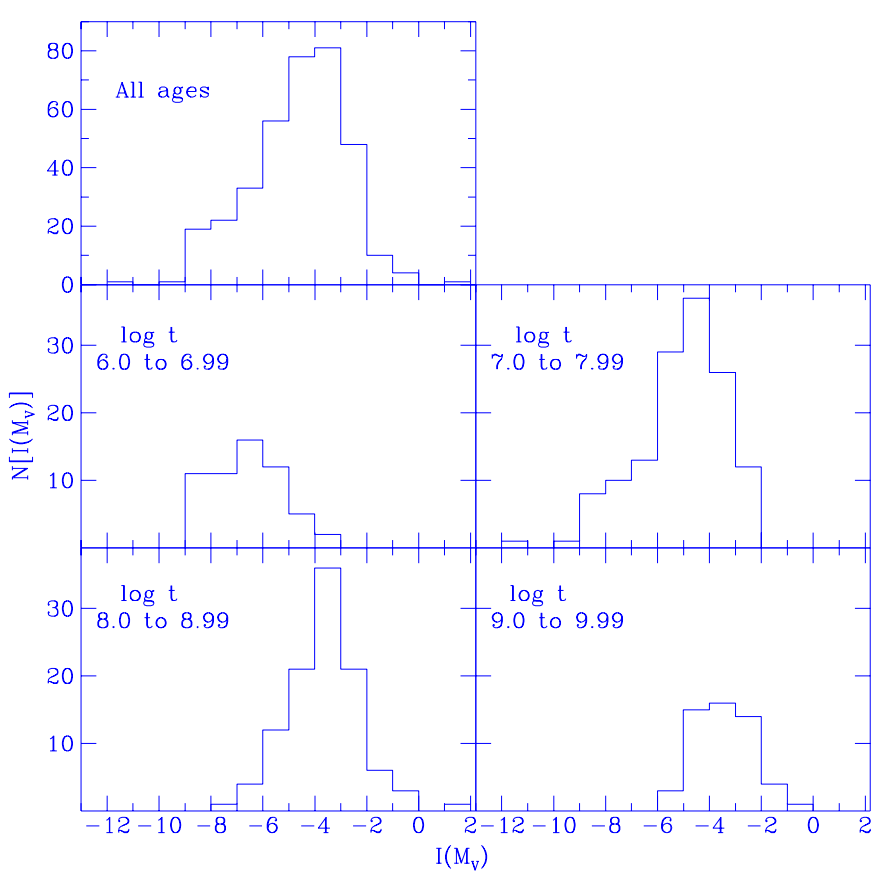

Fig. 4. The histograms are the frequency distribution of the luminosity of 352 clusters according to their ages.

probably due to selection effects while Bhatt et al. (1991) concluded that the observed distribution of open clusters fainter than $I\left(M_{V}\right)=-2.0$ mag may not be entirely due to selection effects. There may be some physical causes of the cut-off at $I\left(M_{V}\right)=-2.0 \mathrm{mag}$. It is important to consider the limiting number of stars to form a cluster because if a multiple-star system with five components is also defined as a cluster then one can expect that the number of clusters fainter than $I\left(M_{V}\right)=-2.0 \mathrm{mag}$ could be large. Although the star formation efficiency is maximum in the low-mass $\left(\sim 100 M_{\odot}\right)$ molecular clouds (Pandey et al. 1990), it is also true that low-mass clouds generate poor clusters only. The lifetime of star clusters depends on the richness of the clusters (Pandey \& Mahra 1986;
Janes \& Adler 1982). Poor clusters have a shorter lifetime compared to the rich ones and very poor clusters (say 5 to 10 stars only) will be disrupted on a very small time scale and consequently, it seems that these will not contribute in any way to the statistics at fainter limits.

From the luminosity distribution for different age groups shown in Fig. 4, one can see that young clusters and the majority of older clusters have integrated magnitudes brighter than $I\left(M_{V}\right)=-2.0 \mathrm{mag}$, while a few old clusters have magnitudes fainter than that. The reason is that with age, dynamical evolution produces mass segregation in a cluster while stellar evolution depletes massive stars (Nilakshi et al. 2002 and references therein). The old clusters which have magnitudes brighter than $I\left(M_{V}\right)=-2.0$ mag most probably contain a number of red giants/supergiants.

\section{Surface density and data completeness}

The surface density of open clusters in the galactic plane, $\sigma$, has been obtained using the relation

$\sigma=N / \pi r^{2}$

where, $N$ is the number of open clusters observed within the completeness radius $r$, for each magnitude interval. The cluster population $N$ and the surface density have also been tabulated in Table 2 for different magnitude intervals. A plot of $\log N$ against $\log d$ (where $d$ is the distance of the cluster) for different integrated brightness is shown in Fig. 5.

Data completeness has been estimated assuming that $\log N \propto \log d$ as suggested by Bhatt et al. (1991). Table 2 and Fig. 5 show that present sample can be considered complete up to $\sim 3.0 \mathrm{kpc}$ whereas the data used by van den Bergh \& Lafontaine (1984) and Bhatt et al. (1991), were complete only up to $\sim 400 \mathrm{pc}$ and $\sim 2.0 \mathrm{kpc}$ respectively. 


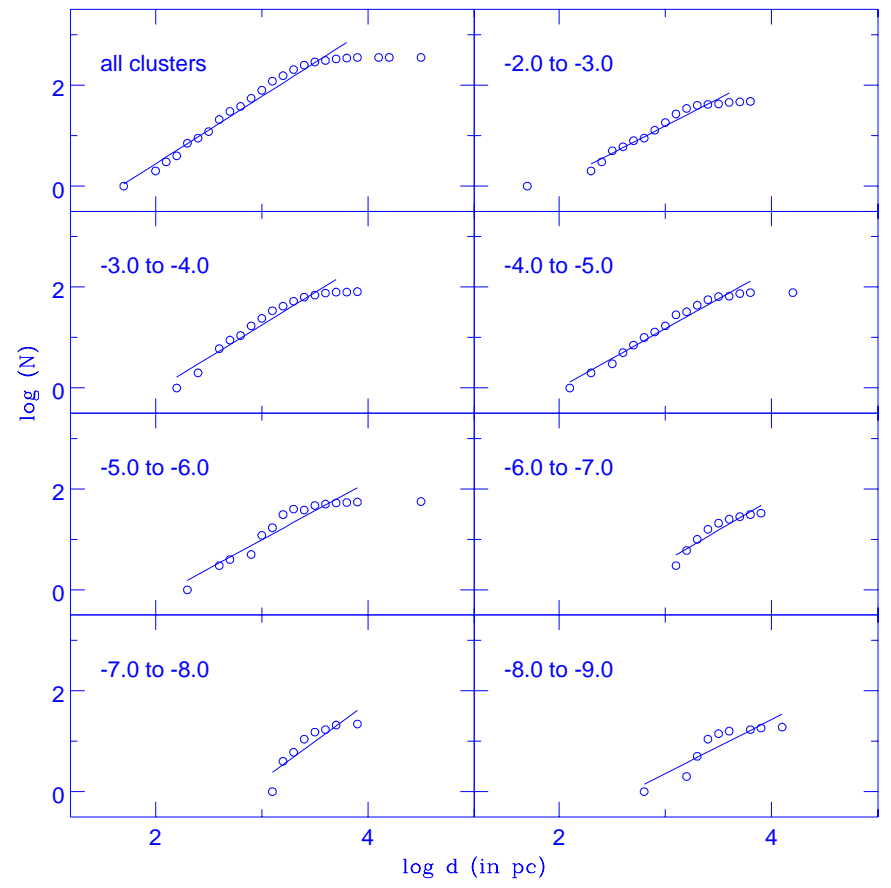

Fig. 5. The $\log N$ versus $\log d$ plots for different integrated magnitude intervals.

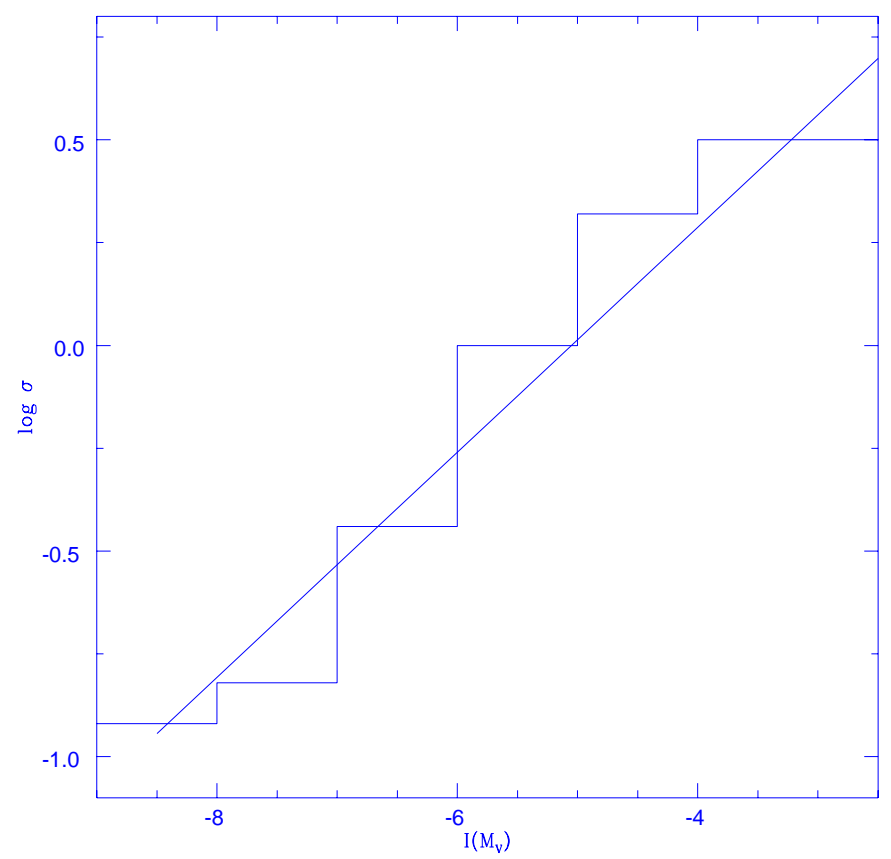

Fig. 6. The integrated luminosity function of the open clusters.

The integrated luminosity function of open clusters brighter than $-2.0 \mathrm{mag}$ is shown in Fig. 6 and can be represented by the relation

$\log \sigma\left(I\left(M_{V}\right)\right)=(0.27 \pm 0.03) I\left(M_{V}\right)+(1.38 \pm 0.17)$

which is in good agreement with the relation obtained by Bhatt et al. (1991). Extrapolation of this equation to higher luminosities, and assuming that the galactic disc has an area of $500 \mathrm{kpc}^{2}$, yields approximately 10 clusters with $I\left(M_{V}\right)=-11.0 \mathrm{mag}$. The luminosity function by
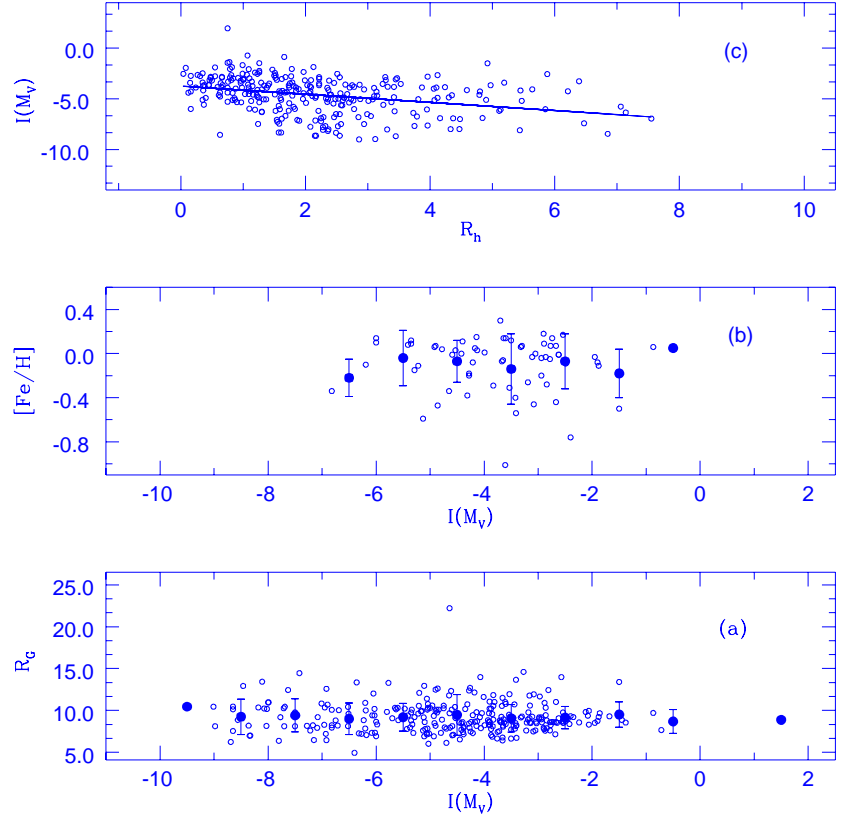

Fig. 7. The galactocentric distance $R_{\mathrm{G}}, I\left(M_{V}\right)$; the metallicity $[\mathrm{Fe} / \mathrm{H}], I\left(M_{V}\right)$ and the heliocentric distance $R_{\mathrm{h}}, I\left(M_{V}\right)$ plots are shown in a), b) and c) panels respectively. The filled circles and error bars in a) and $\mathbf{b}$ ) are the average and $\sigma$ respectively of the data points binned in $I\left(M_{V}\right)$.

van den Bergh \& Lafontaine (1984) yields $10^{2}$ clusters with $I\left(M_{V}\right)=-11.0 \mathrm{mag}$ in the same area. This means that according to the slope of luminosity function obtained in the present analysis only about $1 \%$ of the clusters in the galactic disc have a magnitude of $-11.0 \mathrm{mag}$ while this is about $10 \%$ according to the luminosity function obtained by van den Bergh \& Lafontaine (1984). The disagreement in the slope of the luminosity function obtained in the two studies may be due to large sample, towards the fainter end of the luminosity distribution, used in the present analysis. The present results should therefore be considered more realistic.

\section{Relationship with galactocentric distance, metallicity and heliocentric distance}

Figures $7 \mathrm{a}$ and $\mathrm{b}$ show the variation of $I\left(M_{V}\right)$ with galactocentric distance, $R_{\mathrm{G}}$ (in $\mathrm{kpc}$ ) and metallicity, $[\mathrm{Fe} / \mathrm{H}]$, respectively. They indicate that the $I\left(M_{V}\right)$ does neither depend on $R_{\mathrm{G}}$ nor on $[\mathrm{Fe} / \mathrm{H}]$.

Figures $7 \mathrm{c}$ shows the variation of $I\left(M_{V}\right)$ with heliocentric distance $R_{\mathrm{h}}$ of the clusters. Like Barkhatova \& Pylskaya (1983) and Balázs (1986) the relation between $R_{\mathrm{h}}$ and $I\left(M_{V}\right)$ can be represented by the following equation

$I\left(M_{V}\right)=(-0.40 \pm 0.08) R_{\mathrm{h}}+(-3.76 \pm 0.23)$

where $R_{\mathrm{h}}$ is in kpc. Balázs (1986) found similar effects but he suggests that this correlation is simply caused by selection effects. 


\section{Conclusions}

The integrated magnitudes and colours of 140 open clusters have been derived. Along with results published earlier they have been used to study the characteristics of integrated parameters of galactic star clusters. The main conclusions are:

1. The comparison of observed dependence of integrated luminosity on the age indicates that a steeper MF is needed to match the integrated luminosities of oldest clusters.

2. The integrated luminosity function can be represented by the relation

$$
\log \sigma\left(I\left(M_{V}\right)\right)=(0.27 \pm 0.03) I\left(M_{V}\right)+(1.38 \pm 0.17) .
$$

This indicates that about $1 \%$ of the open clusters in the galactic disc is brighter than $I\left(M_{V}\right)=-11$ mag.

Acknowledgements. We gratefully acknowledge the suggestions given by Prof K. S. de Boer, which improved the contents of the paper.

\section{References}

Balázs, B. A. 1986, Publ. Astron. Dep. Eötvös Univ., No. 8, 41

Barkhatova, K. A., \& Pylskaya, O. P. 1983, Publ. Astrophys. Inst. Czech. Acad. Sci., No. 56, 14

Battinelli, P., Brandimarti, A., \& Capuzzo-Dolcetta, R. 1994, A\&AS, 104, 379

Battinelli, P., \& Capuzzo-Dolcetta, R. 1989, ApJ, 347, 794
Bertelli, G., Bressan, A., Chiosi, C., Fagotto, F., \& Nasi, E. 1994, A\&AS, 106, 275

Bhatt, B. C., Pandey, A. K., \& Mahra, H. S. 1991, JAA, 12, 179

Brocato, E., Castellani, V., Raimondo, G., \& Romaniello, M. 1999, A\&AS, 136, 65

Elson, R. A. W., \& Fall, S. M. 1985, ApJ, 299, 211

Gray, D. F. 1965, AJ, 70, 362

Gray, D. F. 1967, AJ, 72, 800

Janes, K. A., \& Adler, D. 1982, ApJS, 49, 425

Lyngå, G. 1982, A\&A, 109, 213

Maraston, C. 1998, MNRAS, 300, 872

Mermilliod, J.-C. 1995, in Information and on line data in Astronomy, ed. J.-C. Egret, \& M. A. Abrecht (Kulwar Academic Press), 277

Nilakshi, Sagar, R., Pandey, A. K., \& Mohan, V. 2002, A\&A, 383,153

Pandey, A. K., \& Mahra, H. S. 1986, Ap\&SS, 126, 167

Pandey, A. K., Bhatt, B. C., Mahra, H. S., \& Sagar, R. 1989, MNRAS, 236, 263

Pandey, A. K., Paliwal, D. C., \& Mahra, H. S. 1990, ApJ, 362, 165

Piskunov, A. E. 1972, Nauch. Inf. Astr. Counc. Acad. Sci. USSR, 23, 97

Piskunov, A. E. 1974, Nauch. Inf. Astr. Counc. Acad. Sci. USSR, 33, 101

Sagar, R., Joshi, U. C., \& Sinvhal, S. D. 1983, BASI, 11, 44

Schmidt-Kaler, Th. 1967, AJ, 72, 526

Schmidt-Kaler, Th. 1982, Landolt-Bornstein, Numerical data and Funct. Relationship in Sci. and Tech. New Ser., Group 6, vol. 2b, 1

Spassova, N. M., \& Baev, P. V 1985, Ap\&SS, 112, 111

van den Bergh, S., \& Lafontaine, A. 1984, AJ, 89, 1822 